\title{
Phase II, randomized trial of preoperative epirubicin- paclitaxel $+/-$ gefitinib with biomarker evaluation in operable breast cancer
}

\author{
Valentina Guarneri · Antonio Frassoldati - Guido Ficarra · Fabio Puglisi · Claudia Andreetta • \\ Andrea Michelotti · Nicola Cresti · Corrado Boni - Giancarlo Bisagni · Rossana Berardi · \\ Nicola Battelli · Armando Santoro · Giuseppe Banna - Alberto Bottini - Beatrice Di Blasio · \\ Antonino Maiorana $\cdot$ Federico Piacentini $\cdot$ Simona Giovannelli $\cdot$ Gordana Jovic $\cdot$ PierFranco Conte
}

Received: 4 July 2007/ Accepted: 10 July 2007/Published online: 9 August 2007

(C) Springer Science+Business Media B.V. 2007

\begin{abstract}
Purpose To evaluate the in vivo effect of adding gefitinib to preoperative chemotherapy on the EGFR-dependent p42/44 MAPK in operable breast cancer (BC) patients. Secondary aims: to evaluate EGFR, (p)-EGFR, Ki67, apoptotic index (TUNEL test) and VEGFR2 expression from baseline to surgery, percentage of pathologic complete response ( $\mathrm{pCR})$, and toxicity.

Patients and Methods 90 patients with stage II-IIIA BC have been randomized to receive epirubicin $90 \mathrm{mg} / \mathrm{sqm}$ and paclitaxel $175 \mathrm{mg} / \mathrm{sqm}$ on day 1 plus: gefitinib $250 \mathrm{mg}$ daily from day 5 to 16 (Arm A, intermittent), gefitinib $250 \mathrm{mg}$ daily from day 1 to 21 (Arm B, continuous), or placebo (Arm C). Treatment plan: 4 courses every 3 weeks, followed by surgery. Results After preoperative therapy, 86/90 patients underwent surgery; 46 patients $(51 \%)$ received breast conservative surgery. A pCR was observed in 4 patients. No significant differences in the expression of p42/44 MAPK, EGFR,
\end{abstract}

Data presented in part at the 28th Annual San Antonio Breast Cancer Symposium, San Antonio, TX, 8-12 December 2005, and at the 31 st ESMO congress, Istanbul, Turkey, 28 September-3 October 2006

V. Guarneri $(\varangle) \cdot$ A. Frassoldati $\cdot$ F. Piacentini

S. Giovannelli · G. Jovic · PierFrancoConte

Department of Oncology and Hematology,

Modena University Hospital, via del Pozzo

71, 41100 Modena, Italy

e-mail: guarneri.valentina@unimore.it

G. Ficarra · A. Maiorana

Department of Pathology, Modena

University Hospital, Modena, Italy

F. Puglisi · C. Andreetta

Department of Oncology, Udine University Hospital,

Udine, Italy
(p)-EGFR, VEGFR2, proliferation index and apoptosis were observed comparing the combined Arms A + B vs C, and comparing Arm A vs B. Hematologic toxicities were not significantly different comparing Arms A + B vs Arm C, and comparing Arm A vs B. Significantly higher skin and mucosal toxicities were observed when comparing the two gefitinib Arms (A + B) vs Arm C (32\% vs 9.6\%, $P=0.018$; $57 \%$ vs $29 \%, P=0.009$ respectively), while no significant differences were observed comparing Arm A vs B.

Conclusion Adding gefitinib to chemotherapy did not result in different effects on the EGFR-dependent pathway, proliferation, apoptosis and VEGFR2 expression as compared to placebo, while enhancing skin and mucosal toxicity. The two schedules of gefitinib (intermittent vs continuous) did not result in different biologic effects.

Keywords Breast cancer - Primary systemic therapy · Gefitinib · MAPK · Chemotherapy

A. Michelotti · N. Cresti

Division of Oncology, S. Chiara University Hospital, Pisa, Italy

C. Boni · G. Bisagni

Division of Oncology, Arcispedale Santa Maria Nuova,

Reggio Emilia, Italy

R. Berardi · N. Battelli

Division of Oncology, Ancona University Hospital,

Ancona, Italy

A. Santoro · G. Banna

Division of Oncology, Istituto Clinico Humanitas,

Rozzano, Italy

A. Bottini

Division of Oncology, Istituti Ospitalieri, Cremona, Italy

B. D. Blasio

Division of Oncology, Parma University Hospital, Parma, Italy 


\section{Introduction}

The epidermal growth factor receptor (EGFR) is a transmembrane tyrosine kinase receptor of the ErbB family. The aberrant activation of this receptor leads to increased proliferation and angiogenesis, and reduced apoptosis [1]. EGFR is expressed by several epithelial tumors, and in breast cancer the positivity rate ranges between 14 to $91 \%$ [2]. The overexpression of EGFR has been associated with more aggressive breast cancer phenotype, and poorer patient outcome. In contrast to the other ErbB family member HER2, a standardized assay to determine the EGFR status has not been developed, neither a predictive marker for response to anti-EGFR therapies. Moreover, besides the EGFR over-expression, the activation of the EGFR signaling pathway can be due to different mechanisms including the over-expression of EGFR ligands, the mutation of EGFR which results in a constitutively activated form of EGFR, or the heterodimerization with other members of the ErbB family [3-6]. Therefore, the sole measurement of the EGFR expression does not completely encompass the possible role of the EGFR pathway on tumor aggressiveness.

Gefitinib is an orally available, low molecular weight, reversible tyrosine kinase inhibitor of the EGFR, that has documented activity in NSCLC [7-9]. In this disease, the activity of this agent is dependent from the tridimensional structure of the EGFR ATP cleft more than its level of expression [10].

Gefitinib has been tested also in advanced breast cancer both in monotherapy, and in combination with chemotherapy, with limited activity [11-14].

However, early trials with targeted therapies have shown that lack of activity in advanced stages and in heavily pretreated patients may not predict the efficacy in earlier stages of the disease. In fact, these agents predominently act by inhibiting cell proliferation instead of inducing apoptosis, therefore their maximum therapeutic effect might occur in the presence of low tumoral burden.

Primary systemic therapy, besides the proven advantage of increasing the chance for breast-conservative surgery, is the ideal model to test the activity and the efficacy of molecularly targeted agents. In fact, by evaluating the expression of tissue biomarkers before and after treatment, this strategy offers the unique opportunity to directly test in vivo the interactions between drugs and the tumor microenvironment, and their potential relations with tumor response and patient outcome.

We have therefore designed a randomized phase II multicentric trial with gefitinib plus chemotherapy as preoperative treatment for operable breast cancer.

\section{Methods}

\section{Patient selection}

Patients with histologically proven, previously untreated, primary breast cancer were eligible for this study if they met the following criteria: stage II-IIIA (tumor size $>2$ $\mathrm{cm})$ as determined by physical examination and mammography; age 18-70 years; normal cardiac function determined by electrocardiogram and left ventricular ejection fraction (L-VEF); World Health Organization (WHO) performance status 0 ; adequate contraceptive method (women of childbearing potential). The exclusion criteria were: locally advanced disease (stage IIIB) or inflammatory breast carcinoma; active infection; presence of distant metastases; any evidence of clinically active interstitial lung disease; absolute neutrophil count (ANC) less than $1.5 \times 10^{9} / 1$, or platelets less than $100 \times 10^{9} / 1$; serum bilirubin greater than 1.5 times the upper limit of reference range (ULRR); alanine amino transferase (ALT) or aspartate amino transferase (AST) greater than 2.5 times the ULRR; any evidence of severe or uncontrolled systemic disease; concomitant use of phenytoin, carbamazepine, rifampicin, barbiturates, or St John's Wort; pregnancy or breastfeeding.

Pre-study staging included mammography, chest XR, bone scan, liver ultrasonography, echocardiography and hematological blood tests.

The local human investigation committees of participating institutions approved the protocol; all patients gave the written informed consent to participate the study.

Study design

At baseline, a large core (14 gauge) breast biopsy for histological diagnosis and for the immunohistochemical (IHC) analyses of tumor biomarkers was performed.

Eligible patients were therefore randomized to receive chemotherapy with epirubicin $90 \mathrm{mg} / \mathrm{m}^{2}$ intravenous (iv) bolus plus paclitaxel $175 \mathrm{mg} / \mathrm{m}^{2}$ iv infusion over $3 \mathrm{~h}$ on day 1 for 4 courses administered every 3 weeks plus: gefitinib $250 \mathrm{mg}$ orally once daily from day 5 to day 16 of four 3-weekly cycles of chemotherapy (Arm A, intermittent); or gefitinib $250 \mathrm{mg}$ orally once daily from days 1 to 21 for four 3-weekly cycles of chemotherapy (Arm B, continuous); or placebo orally daily for four 3-weekly cycles of chemotherapy (Arm C, control). Arm $\mathrm{B}$ and Arm $\mathrm{C}$ were double blinded. The study plan is showed in Panel 1. Patients underwent surgery within 5 weeks from the administration of the 4th chemotherapy cycle. 
Panel 1 Treatment plan

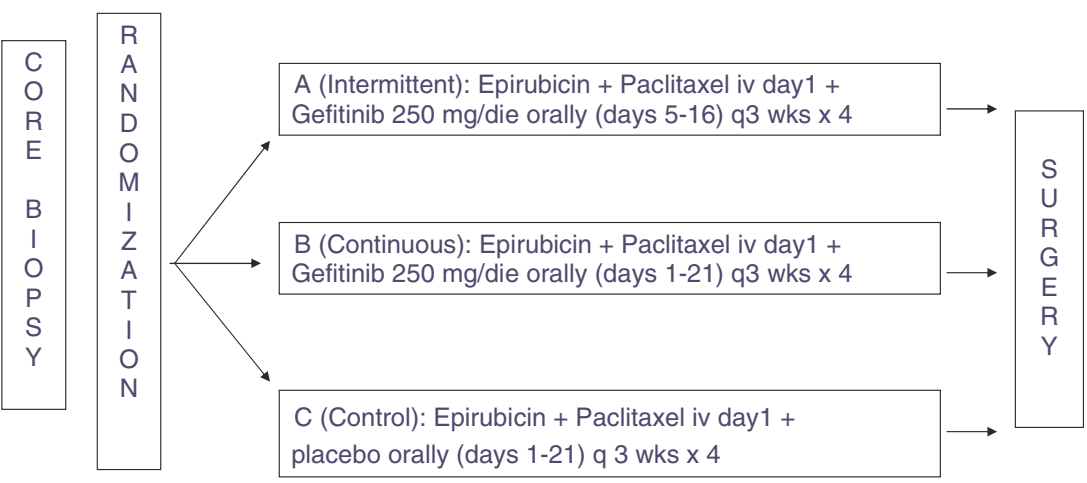

Chemotherapy was not administered in case of: WBC $<3 \times 10^{9} / 1$, ANC $<1.5 \times 10^{9} / 1$, or platelet count was $<100 \times 10^{9} / 1$. Epirubicin and paclitaxel doses were reduced by $25 \%$ in case of: febrile neutropenia, grade 4 thrombocytopenia lasting $>3$ days and/or thrombocytopenia associated with bleeding, grade 4 neutropenia lasting $>7$ days, grade 3 non hematologic toxicities (except for nausea/vomiting and alopecia). In case of grade 4 non hematologic toxicities epirubicin and paclitaxel doses were reduced by $50 \%$ or interrupted. Paclitaxel dose was reduced by $25 \%$ in case of grade 1 neurotoxicity, and discontinued in case of grade 2 neurotoxicity.

No dose reduction for gefitinib was foreseen. Repeated dose interruptions were allowed for a maximum of 14 days in case of: grade 3-4 diarrhea, grade 2 diarrhea with rapidly declining ANC, poorly tolerated skin toxicity, respiratory symptoms suspicious for interstitial lung disease, any other grade 3-4 toxicity or lower clinically relevant toxicity. Gefitinib dose was restarted when toxicity reverted to grade 1 .

Study aims

The primary end-point of the study was to evaluate the inhibition induced by chemotherapy plus gefitinib vs chemotherapy plus placebo on the EGFR dependent p42/44 MAPK from biopsy to surgery. Secondary aims were to evaluate the modifications in EGFR and activated/phosphorylated (p)-EGFR expression, in proliferation index, apoptotic index and VEGF-R (Flk1) expression from biopsy to surgery; to evaluate the percentage of $\mathrm{pCR}$, and the toxicity of this combination. pCR was defined as complete disappearance of invasive carcinoma in both breast and axillary lymph nodes. Residual DCIS was included in the pCR category.

Pathology

All the specimens from both biopsy and surgery were centrally analyzed at the Department of Pathology in Modena for the evaluation of the following parameters: $\mathrm{p} 42 / 44$
MAPK, EGFR, (p)-EGFR, proliferation index (Ki-67), apoptotic index (TUNEL assay) and VEGFR2 (KDR/Flk1) expression. A dedicated breast pathologist, blinded to patient treatment allocation and clinical outcome, reviewed all paired biopsy and surgical specimens.

\section{Immunohistochemical staining}

Tumor specimens has been fixed in $10 \%$ neutral-buffered formalin for 20-28 h before processing and embedding at local pathology centers in paraffin wax blocks.

The antibody used were Phospho-p44/42 MAP Kinase (Thr202/Tyr204), Cell Signaling, diluited at 1:30; (p)-EGFR (Tyr 1068), Cell Signaling, diluted at 1:20; EGFR clone 31G7, Zymed, diluted at 1:100; VEGFR2 (FLK1), Neomarkers, diluted at 1:150; Ki 67clone Ki-mib-1 diluted at 1:200. Immunohistochemical staining was performed according to the avidin-biotin method, using tissue sections of 3 micron thickness. After deparaffinization in xylene and graded alcohols, epitope retrieval was performed. Antigene retrieval for p42/44 MAPK, (p)-EGFR, VEGFR2 and Ki-67 was made in 10 mMEDTA buffer ( $\mathrm{pH} 8$ ) in a microwave. Epitope retrieval for EGFR was made by protease XIV for $10 \mathrm{~min}$. After epitope retrieval, endogenous peroxidase was blocked by $0.3 \%$ hydrogen peroxide for $15 \mathrm{~min}$. Section were incubated with primary antibody for $30 \mathrm{~min}$ at $37^{\circ} \mathrm{C}$. then with the biotinylated secondary antibody for $20 \mathrm{~min}$ at $37^{\circ} \mathrm{C}$ and then in avidin-biotin complex for a further $45 \mathrm{~min}$. Diaminobenzidine tetrahydrochloride (DAB) was used as chromogen. The following parameters has been recorded: presence or absence of immunoreaction (diffuse or focal), cell types exhibiting a positive reaction (tumor cells, endothelia, stromal cells, inflammatory cells) and percentage of immunostained cells.

\section{Assessment of the apoptotic index}

The sections were stained for apoptosis using the terminal deoxynucleotidyl transferase biotin-dUTP nick end labeling technique. Apoptotic cells were identified by the 
TUNEL method, according the standard procedure included in the Apop Tag Plus In situ Apoptosis Detection Kit (Chemicon International). The Apoptotic Index (AI) was defined as the percentage of apoptotic events per cells population. The AI was assessed by counting at least 3,000 malignant cells randomly selected at $400 \times$ magnification.

\section{Statistics}

The primary comparison was planned between combined arms $\mathrm{A}$ and $\mathrm{B}$ versus arm C. Assuming the mean percentage of p42/44 MAPK positive cancer cells $x \%$ after four courses of chemotherapy and placebo, and Y\% after four courses of chemotherapy plus gefitinib (intermittent and continuous arms combined), 30 subjects per treatment arm would be sufficient to detect a reduction to a $y \%$ of half of $\mathrm{x} \%$ at a two sided alpha of $5 \%$ with a power of $80 \%$, and provided the standard deviation of the difference preand post-gefitinib was no more than 0.7 times $\mathrm{x} \%$, assuming a drop out rate of $1 / 6$ and making an allowance for non-parametric test.

The Wilcoxon rank sum test was used to compare median differences of biomarker values from baseline to surgery in Arms A and B pooled and Arm C. All subjects that were randomised and received the first dose of study treatment were considered intention-to-treat (ITT) population. Safety analysis set is composed of all patients who received al least the first dose of study treatment (all 90 patients). Chi-square test was used to compare the proportions of patients with various types of toxicity.

The Wilcoxon sign rank matched pairs test was used to evaluate differences between baseline and surgery biomarker expression.

\section{Results}

Patients and response

From April 2004 to January 2006, 90 patients from 10 Italian Centers were randomized. Table 1 summarizes patient characteristics overall, and by treatment arm.

The median age of the whole group was 51 (range 29-69). The clinical stage was IIA in 36 patients (40\%), IIB in 44 patients (49\%), and IIIA in 9 patients (10\%). One patient with clinical stage IIIB was wrongly enrolled but was considered for all the protocol outcomes according to the ITT analysis. Patients characteristics were balanced among the 3 treatment arms, with the exception of HER2 and EGFR expression.

Eighty-six out of 90 patients have been submitted to surgery after primary treatment; reasons for not undergoing surgery were: one death because of car accident, 1 patient major protocol deviation and lost to follow up, 1 treatment

Table 1 Patient characteristics overall, and by treatment arm (\% of patients)

\begin{tabular}{|c|c|c|c|c|}
\hline & ALL & ARM A & ARM B & ARM C \\
\hline Number & 90 & 27 & 32 & 31 \\
\hline Median age, years (range) & $51(29-69)$ & $51(29-68)$ & $46.5(33-69)$ & $51(31-66)$ \\
\hline Mean $T$ size with breast imaging, $\mathrm{cm}$ (range) & $3.3(2-9)$ & $3.2(2-9)$ & $3.3(2-6)$ & $3.6(2-7.5)$ \\
\hline \multicolumn{5}{|l|}{ Clinical stage* } \\
\hline IIA & 40 & 48.2 & 34.4 & 38.7 \\
\hline IIB & 49 & 37 & 56,2 & 51.6 \\
\hline IIIA & 10 & 14.8 & 9.4 & 6.5 \\
\hline \multicolumn{5}{|l|}{ Histologic type } \\
\hline Ductal & 81.1 & 85.2 & 84.4 & 74.2 \\
\hline Lobular & 5.5 & 0 & 6.2 & 9.7 \\
\hline Other/NA & 13.3 & 14.8 & 9.4 & 16.1 \\
\hline \multicolumn{5}{|l|}{ Histologic Grade } \\
\hline G2 & 30 & 37 & 22 & 32 \\
\hline G3 & 50 & 52 & 56 & 42 \\
\hline NA & 20 & 11 & 22 & 26 \\
\hline ER positivity & 70 & 74 & 64 & 72 \\
\hline PgR positivity & 52 & 52 & 42 & 60 \\
\hline HER2+ & 24 & 30 & 31 & 10 \\
\hline EGFR $>/=10 \%$ & 14 & 4 & 11 & 27 \\
\hline
\end{tabular}

* 1 pt stage IIIB in Arm C 
interruption due to adverse event, and one death due to myocardial infarction in the operating room.

According to ITT analysis, 46/90 patients (51\%) underwent breast conservative surgery: 17 (63\%) in Arm A, $15(47 \%)$ in Arm B, and 14 (45\%) in Arm C. A pCR in both breast and axillary nodes was observed in 4 patients: 2 in Arm A, 1 in Arm B and C respectively. The pCR rate was significantly higher in hormone receptor negative versus positive tumors $(12.5 \%$ vs $1.5 \%, P=0.02)$. No significant differences were observed according to HER2 status (pCR rate $9.5 \%$ in HER2+ vs $3.2 \%$ in HER2-, $P=0.25$ ) or to EGFR expression (pCR rate $6.6 \%$ in EGFR+ vs $4.3 \%$ in EGFR,$- P=0.70$ ).

Compliance with treatment and toxicity

Overall, 10 patients discontinued the experimental treatment: 1 patient in Arm A for a grade 3 hypertransaminasemia, 4 patients in Arm B (1 treatment and cancer unrelated death; 1 grade 3 hypertransaminasemia; 1 progression of disease; 1 grade 3 skin toxicity), and 5 patients in Arm C (1 grade 2 enteritis; 1 grade 4 hypertransaminasemia; 1 perforative peritonitis; 1 severe mood alteration requiring carbamazepine; 1 major protocol deviation).

Chemotherapy dose reductions or delays were necessary in $28 \%$ of courses in Arm A, 19\% in Arm B and 15\% in Arm C $(P=\mathrm{ns})$.

Grade 3-4 hematologic toxicities per treatment arms are summarized in Table 2: no significant differences were observed when comparing the two arms with chemotherapy plus gefitinib (Arms A and B) versus chemotherapy plus placebo (Arm C), nor comparing the two different schedules of gefitinib in combination with chemotherapy (Arm A vs Arm B).

Non hematologic toxicities are summarized in Table 3. Significantly higher skin and mucosal toxicities were observed when comparing the two gefitinib Arms $(\mathrm{A}+\mathrm{B})$ vs the placebo arm $\mathrm{C}(32.2 \%$ vs $9.6 \%, P=0.018$ and $57.6 \%$ vs $29.0 \%, P=0.009$ respectively). Again, no significant differences were observed when comparing Arm A vs Arm B (intermittent vs continuous gefitinib).

\section{Biomarker expression}

Adding gefitinib to chemotherapy did not result in a decreased expression of p42/44 MAPK from baseline to surgery as compared to chemotherapy plus placebo: the median (min;max) differences in $\mathrm{p} 42 / 44$ MAPK expression were $5(-70 ; 90)$ in combined Arms A + B and $5(-14$; $52)$ in $\operatorname{Arm} C(P=0.98)$. The same observation was found when comparing Arm A vs Arm B: the median (min;max) differences were $6.5(-2 ; 60)$ and $3(-70 ; 50)$ respectively $(P=0.4870)$ (Fig. 1).

Similar findings were observed in the expression of the other tumor biomarkers (EGFR, (p)-EGFR, VEGFR2, proliferation and apoptosis). No significant differences were observed comparing the combined Arms A + B vs C, and comparing Arm A vs B (Table 4).

On the contrary, irrespectively of treatment arm, a significant reduction in the expression of tumor biomarkers from baseline to surgery was observed, with the exception of EGFR. In particular, the median (min;max) values at baseline and at surgery were: p42/44 MAPK $7(0 ; 65)$ and 1 $(0 ; 70) \quad(P<0.001)$; (p)-EGFR $0 \quad(0 ; 40)$ and $0(0 ; 20)$ $(P=0.0019)$; Ki $6730(3 ; 90)$ and $15(1 ; 90)(P<0.0001)$; apoptotic index $1.105(0.05 ; 4.7)$ and $0.71 \quad(0.1 ; 4.3)$ $(P=0.0007)$; VEGFR2 $2(0 ; 80)$ and $0(0 ; 20)(P<0.001)$. The data for p42/44 MAPK, apoptotic index, Ki 67 and VEGFR2 are represented in Fig. 2.

\section{Discussion}

Primary systemic therapy for operable disease represents the ideal model to test the in vivo effects of targeted agents on the tumor microenvironment. In fact, the diagnostic biopsy allows for the evaluation of unperturbed tumor characteristics, while the measurement of the same

Table 2 Grade 3-4 Hematologic toxicity by treatment arm (Number and \% of patients)

\begin{tabular}{|c|c|c|c|c|c|c|}
\hline & \multicolumn{2}{|c|}{ ARM A (27 pts) } & \multicolumn{2}{|c|}{ ARM B (32 pts) } & \multicolumn{2}{|c|}{ ARM C (31 pts) } \\
\hline & $\begin{array}{l}\text { G3 } \\
N(\%)\end{array}$ & $\begin{array}{l}\mathrm{G} 4 \\
N(\%)\end{array}$ & $\begin{array}{l}\text { G3 } \\
N(\%)\end{array}$ & $\begin{array}{l}\mathrm{G} 4 \\
N(\%)\end{array}$ & $\begin{array}{l}\text { G3 } \\
N(\%)\end{array}$ & $\begin{array}{l}\mathrm{G} 4 \\
N(\%)\end{array}$ \\
\hline Leukopenia & $1(3.7)$ & - & $1(3.1)$ & - & - & - \\
\hline Neutropenia & $3(11.1)$ & $9(33.3)$ & $7(21.8)$ & $9(28.1)$ & 7 (22.6) & $7(22.6)$ \\
\hline Anemia & - & - & $1(3.1)$ & $1(3.1)$ & - & - \\
\hline Thrombocytopenia & - & - & - & - & - & - \\
\hline Febrile neutropenia & $2(7.4)$ & $1(3.7)$ & $1(3.1)$ & - & $1(3.2)$ & - \\
\hline
\end{tabular}

$\chi^{2}$-test, $P$ value not significant for $\mathrm{A}+\mathrm{B}$ vs $\mathrm{C}$ and $\mathrm{A}$ vs $\mathrm{B}$ 
Table 3 Non-hematologic toxicity by treatment arm (Number and \% of patients)

\begin{tabular}{|c|c|c|c|c|c|c|c|c|c|c|}
\hline & \multicolumn{3}{|c|}{ ARM A (27 pts) } & \multicolumn{3}{|c|}{ ARM B (32 pts) } & \multicolumn{4}{|c|}{ ARM C (31 pts) } \\
\hline & $\begin{array}{l}\mathrm{G} 1 \\
N(\%)\end{array}$ & $\begin{array}{l}\mathrm{G} 2 \\
N(\%)\end{array}$ & $\begin{array}{l}\mathrm{G} 3 \\
N(\%)\end{array}$ & $\begin{array}{l}\text { G1 } \\
N(\%)\end{array}$ & $\begin{array}{l}\mathrm{G} 2 \\
N(\%)\end{array}$ & $\begin{array}{l}\text { G3 } \\
N(\%)\end{array}$ & $\begin{array}{l}\mathrm{G} 1 \\
N(\%)\end{array}$ & $\begin{array}{l}\mathrm{G} 2 \\
N(\%)\end{array}$ & $\begin{array}{l}\mathrm{G} 3 \\
N(\%)\end{array}$ & $\begin{array}{l}\mathrm{G} 4 \\
N(\%)\end{array}$ \\
\hline Nausea/Vomiting & $13(48.1)$ & $6(22.2)$ & $2(7.4)$ & $12(37.5)$ & $8(25)$ & - & $13(41.9)$ & $9(29.0)$ & $1(3.2)$ & - \\
\hline Mucositis* & $11(40.7)$ & $6(22.2)$ & - & $10(31.2)$ & $6(18.7)$ & $1(3.1)$ & $4(12.9)$ & $5(16.1)$ & - & - \\
\hline Diarrhoea & $4(14.8)$ & $3(11.1)$ & $1(3.7)$ & $6(18.7)$ & $5(15.6)$ & $1(3.1)$ & $2(6.4)$ & $3(9.6)$ & $2(6.4)$ & - \\
\hline Alopecia & $1(3.7)$ & $5(18.5)$ & $1(3.7)$ & $3(9.3)$ & $7(21.8)$ & $3(9.3)$ & $4(12.9)$ & $9(29.0)$ & $3(9.6)$ & - \\
\hline Hepatotoxicity & $1(3.7)$ & - & $3(11.1)$ & - & $2(6.2)$ & $2(6.2)$ & $1(3.2)$ & - & $1(3.2)$ & $1(3.2)$ \\
\hline Neurotoxicity & $6(22.2)$ & - & $1(3.7)$ & $7(21.8)$ & - & - & $8(25.8)$ & $3(9.6)$ & - & - \\
\hline Skin toxicity** & 7 (25.9) & $2(7.4)$ & - & $2(6.2)$ & $7(21.8)$ & $1(3.1)$ & $3(9.6)$ & - & - & - \\
\hline Allergic reaction & $1(3.7)$ & $1(3.7)$ & - & $1(3.1)$ & $2(6.2)$ & $2(6.2)$ & $1(3.2)$ & - & $1(3.2)$ & - \\
\hline Pulmonary & $2(7.4)$ & $1(3.7)$ & - & $5(15.6)$ & $1(3.1)$ & - & $2(6.4)$ & - & - & - \\
\hline
\end{tabular}

* $P=0.009$ Arms A + B vs C

** $P=0.018$ Arms A + B vs C

Fig. 1 Box plots of the differences in $\mathrm{p} 42 / 44$ MAPK expression from baseline to surgery. Panel 1.1: differences from baseline to surgery in arm A (light gray plot) and arm B (grey plot); Wilcoxon rank sum test, $P=0.49$. Panel 1.2:

differences from baseline to surgery in combined arms A and B (light gray plot) and arm C (gray plot); Wilcoxon rank sum test, $P=0.98$
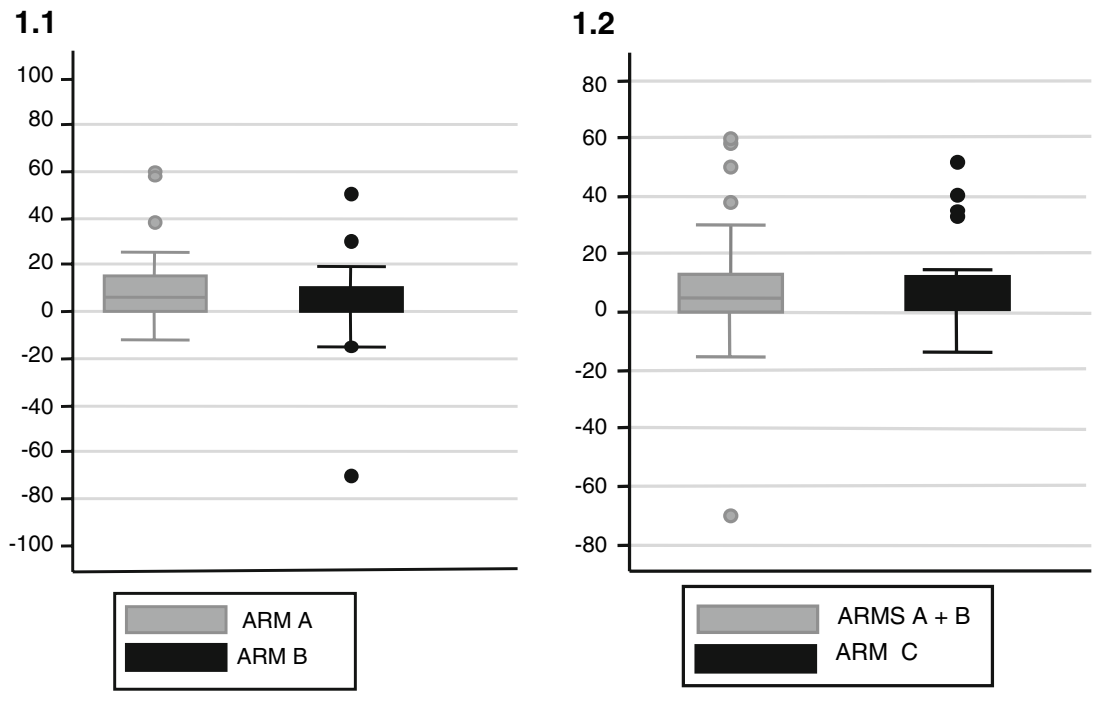

Table 4 Median differences between pre-treatment biopsy and surgery (min, max)

\begin{tabular}{lllllll}
\hline Biological parameter & Arm A & Arm B & Arm A + B & Arm C & $P$-value $(A+B$ vs C) & $P$-value $($ A vs B) \\
\hline p42/44 MAPK & $6.5(-12 ; 60)$ & $3(-70 ; 50)$ & $5(-70 ; 60)$ & $5(-14 ; 52)$ & 0.78 & 0.38 \\
EGFR & $0(0 ; 5)$ & $0(-4 ; 20)$ & $0(-4 ; 20)$ & $0(-20 ; 80)$ & 0.95 & 0.4 \\
(p)-EGFR & $0(0 ; 40)$ & $0(-2 ; 5)$ & $0(-2 ; 40)$ & $0(0 ; 25)$ & 0.49 & 0.23 \\
Ki67 & $1.5(-25 ; 55)$ & $10(-4 ; 65)$ & $6(-25 ; 65)$ & $2.5(-55 ; 63)$ & 0.28 & 0.06 \\
Tunel & $0.47(-3.45 ; 3.16)$ & $0.27(-1.04 ; 2.1)$ & $0.3(-3.45 ; 3.16)$ & $0.31(-3.92 ; 3.6)$ & 1.00 & 0.69 \\
VEGF-R & $1(-2 ; 80)$ & $0(-5 ; 30)$ & $0(-5 ; 80)$ & $0(-3 ; 50)$ & 0.67 & 0.41 \\
\hline
\end{tabular}

biomarkers after therapy gives the opportunity to better understand the interactions between biomarker modulation and treatment effects.

This phase II randomized trial was designed on the basis of a biological primary end point: to evaluate the effect of chemotherapy plus gefitinib vs chemotherapy plus placebo on the expression of the EGFR dependent p42/44 MAPK (also called ERK). The mitogen-activated protein kinase (MAPK) family is an intracellular pathway that regulates cell survival and death. In particular, the stress-activated protein kinases JNK/SAPK and p38MAPK seem to promote apoptotic signaling in response to chemical and 
Fig. 2 Box plots of biomarker expression from baseline (pink plots) to surgery (blue plots); (Wilcoxon signed rank matched pairs test). Panel 2.1: p42/44 MAPK $(P<0.001)$. Panel 2.2: Apoptotic Index (Tunel test) $(P=0.0007)$. Panel 2.3: Ki 67 $(P<0.0001)$. Panel 2.4: VEGFR2 $(P<0.001)$

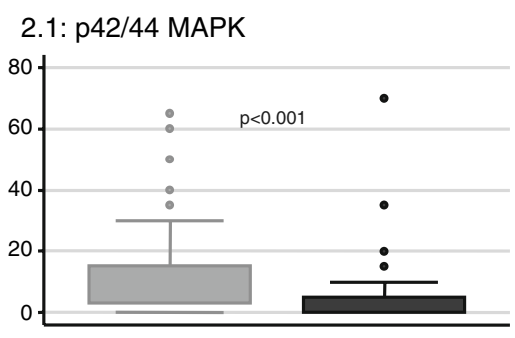

2.2 Apoptotic index (Tunel test)

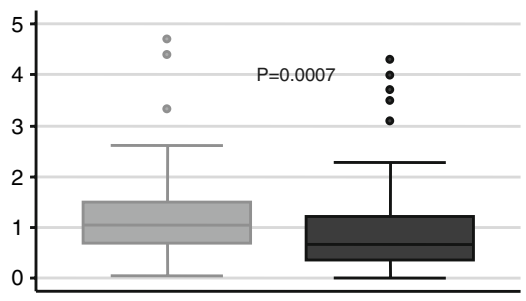

2.3: Ki 67

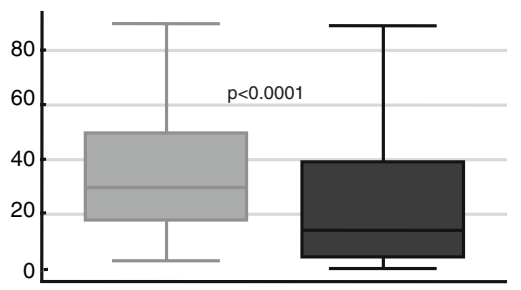

\section{4: VEGFR2}

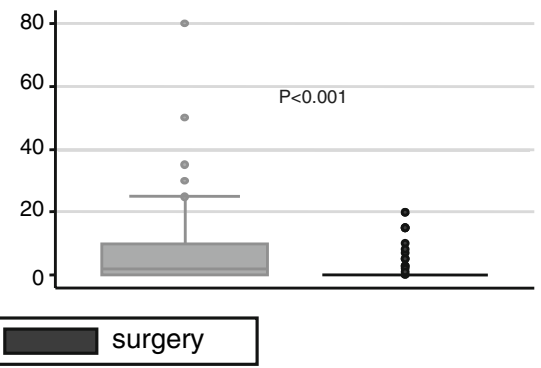

environmental stress. The mitogen activated protein $\mathrm{p} 42 / 44$ MAPK is activated by extracellular signaling, and its activation has been associated with cell proliferation and survival [15-17].

We hypothesized that the addition of the tyrosine kinase inhibitor of EGFR gefitinib to chemotherapy would result in an enhanced inhibition of the p42/44 MAPK as compared to chemotherapy plus placebo. Contrary to this expectation, the inhibition of p42/44 MAPK was not significantly different between patients receiving or not gefitinib. However, a significant inhibition of this intracellular pathway was observed independently by treatment arm. Therefore, we can hypothesize that chemotherapy by itself can inhibit the p42/44 MAPK, thus masking the effect of gefitinib on its specific cellular pathway. The effect of chemotherapy on the p42/44 MAPK has been extensively studied on cell lines, however without conclusive results [18]. In particular, paclitaxel has been shown to increase the p42/44 MAPK activity, with either enhanced or decreased apoptosis, as well as no effect [19-21]. Therefore, whether activation of p42/44 MAPK signaling represents a protective or proapoptotic response to paclitaxel exposure remains an unsolved question. Data with anthracyclines are even more scanty and uncertain [18].

Similarly to that observed for p42/44 MAPK, the expression of EGFR, (p)-EGFR, VEGFR2 as well as tumor proliferation (Ki 67) and apoptotic index was not significantly modified by adding to chemotherapy gefitinib or placebo. On the contrary, except for the EGFR, all these parameters were significantly reduced after therapy, irrespectively of treatment arm.

Our patient population was not selected nor stratified for EGFR status. This choice was dictated by the lack of reproducibility among different laboratories in the EGFR assay and by the uncertain relation between EGFR expression and its dependent signaling pathway in breast cancer [22]. Unfortunately, by chance, in the chemotherapy plus placebo arm there was a significantly lower percentage of HER2-positive and a significantly higher percentage of EGFR-expressing tumors. The most interesting results with gefitinib derive from a randomized trial comparing preoperative gefitinib vs gefitinib plus anastrozole in a patient population selected for EGFR and ER expression [23]. We cannot therefore rule out the hypothesis that our results are influenced by the unselected patient population and the imbalance in HER-family expression in the three arms. However, as a matter of fact, the inhibition of the EGFR dependent pathway, was surprisingly consistent across the different treatment arms.

The pCR rate was a secondary end point of the study and was lower than expected.

It is known that the probability of achieving a pCR is significantly higher in case of lower $\mathrm{T}$ size, and negative hormone receptors. In our study population, the mean $T$ size was $3.3 \mathrm{~cm}$ (range $2-9 \mathrm{~cm}$ ) and $70 \%$ of the patients had ER positive tumors. These features might in part account for the low $\mathrm{pCR}$ rate. In a randomized trial comparing preoperative anastrozole + gefitinib vs anastrozole in operable breast cancer patients, the combination resulted in a lower inhibition of ki67, and in a trend for a lower ORR versus anastrozole alone, thus suggesting a potential detrimental effect of gefitinib [24]. In our trial no difference in the $\mathrm{pCR}$ rate was observed comparing the arms with gefitinib plus chemotherapy versus chemotherapy plus placebo, therefore it is unlike that gefitinib had in some way reduced the activity of chemotherapy. Furthermore, despite the low rate of pCR, we observed an interesting rate of breast conservative surgery (51\%), 
considering that $60 \%$ of the patients were stage IIB or IIIA at diagnosis.

Overall, treatment was well tolerated, and the toxicity profile was not dissimilar to that reported for either treatment alone. As expected, the combination of gefitinib plus chemotherapy (Arms A + B) resulted in a significantly higher skin and mucosal toxicity as compared to chemotherapy plus placebo (Arm C). Unexpectedly, considering that patients in Arm B received almost doubled dose of gefitinib (21 daily doses vs 12 daily doses in Arm A) no differences in toxicity were observed when comparing the two different schedules of gefitinib administration. In conclusion, adding gefitinib to chemotherapy does not result in different effects on the EGFR-dependent pathway, proliferation, apoptotic index and VEGFR2 expression as compared to chemotherapy plus placebo, while enhancing skin and mucosal toxicity. Moreover, the two schedules of gefitinib (intermittent vs continuous) did not result in significantly different biologic effects.

These and other disappointing results obtained both in advanced and in early disease, suggest that the inhibition of the EGFR-driven pathway is unlikely to play a key role in the majority of breast cancers. A better standardization of EGFR assay, a more precise definition of the role of EGFR pathway in specific molecular subsets such as triple negative disease and availability of new ErbB family targeting agents, are likely to renew the interest in targeting this signaling pathway in breast cancer.

Acknowledgments This trial was coordinated and supervised by the principal investigators with funding and organizational support from the trial sponsor AstraZeneca. The corresponding author had full access to all the data in the study and had final responsibility for the decision to submit for publication

\section{References}

1. Salomon DS, Brandt R, Ciardiello F et al (1995) Epidermal growth factor related peptides and their receptors in human malignancy. Crit Rev Haematol 19:183-232

2. Klijn JG, Berns PM, Schmitz PI et al (1992) The clinical significance of epidermal growth factor receptor (EGF-R) in human breast cancer: a review on 5232 patients. Endocr Rev 13:3-17

3. Tang CK, Gong XQ, Moscatello DK et al (2000) Epidermal growth factor receptor vIII enhances tumorigenicity in human breast cancer. Cancer Res 60:3081-3087

4. Khazaie K, Schirrmacher V, Lichtner RB (1993) EGF receptor in neoplasia and metastasis. Cancer Metastasis Rev 12:255-274

5. Messa C, Russo F, Caruso MG et al (1998) EGF, TGF-alpha, and EGF-R in human colorectal adenocarcinoma. Acta Oncol 37:285-289

6. Karunagaran D, Tzahar E, Beerli RR et al (1996) ErbB-2 is a common auxiliary subunit of NDF and EGF receptors: implications for breast cancer. EMBO J 15:254-264
7. Wakeling AE, Guy SP, Woodburn JR et al (2002) ZD1839 (Iressa): An orally active inhibitor of epidermal growth factor signaling with potential for cancer therapy. Cancer Res 62:5749-5754

8. Kris MG, Natale RB, Herbst RS et al (2003) Efficacy of gefitinib, an inhibitor of the epidermal growth factor receptor tyrosine kinase, in symptomatic patients with non-small cell lung cancer: a randomized trial. JAMA 290:2149-2158

9. Fukuoka M, Yano S, Giaccone G et al (2003) Multi-institutional randomized phase II trial of gefitinib for previously treated patients with advanced non-small-cell lung cancer (The IDEAL 1 Trial). J Clin Oncol 21:2237-2246

10. Lynch TJ, Bell DW, Sordella R et al (2004) Activating mutations in the epidermal growth factor receptor underlying responsiveness of non-small-cell lung cancer to gefitinib. N Engl J Med 350:2129-2139

11. Baselga J, Albanell J, Ruiz A et al (2005) Phase II and tumor pharmacodynamic study of gefitinib in patients with advanced breast cancer. J Clin Oncol 23:5323-5333

12. Gasparini G, Sarmiento R, Amici S et al (2005) Gefitinib (ZD1839) combined with weekly epirubicin in patients with metastatic breast cancer: a phase I study with biological correlate. Ann Oncol 16:1867-1873

13. Fountzilas G, Pectasides D, Kalogera-Fountzila A et al (2005) Paclitaxel and carboplatin as first-line chemotherapy combined with gefitinib (IRESSA) in patients with advanced breast cancer: a phase I/II study conducted by the Hellenic Cooperative Oncology Group. Breast Cancer Res Treat 92:1-9

14. Ciardiello F, Troiani T, Caputo F et al (2006) Phase II study of gefitinib in combination with docetaxel as first line therapy in metastatic breast cancer. Br J cancer 94:1604-1609

15. Cobb MH (1999) MAP kinase pathway. Prog Biophys Mol Biol 71:479-500

16. Davis RJ (2000) Signal transduction by the JNK group of MAP kinases. Cell 103:239-252

17. Ono K, Han J (2000) The p38 signal transduction pathway: activation and function. Cell Signal 12:1-13

18. Fan M, Chambers TC (2001) Role of mitogen-activated protein kinases in the response of tumor cells to chemotherapy. Drug Resist Updat 4:253-267

19. Shtil AA, Mandlekar S, Yu R et al (1999) Differential regulation of mitogen-activated protein kinases by microtubule-binding agents in human breast cancer cells. Oncogene; 18:377-384

20. Huang Y, Sheikh MS, Fornace AJ et al (1999) Serine protease inhibitor TPCK prevents Taxol-induced cell death and blocks cRaf-1 and Bcl-2 phosphorylation in human breast carcinoma cells. Oncogene 18:3431-3439

21. Bacus SS, Gudkov AV, Lowe M et al (2001) Taxol-induced apoptosis depends on MAP kinase pathway (ERK and p38) and is independent of p53. Oncogene 20:147-155

22. Chan SK, Hill ME, Gullick WJ (2006) The role of the epidermal growth factor receptor in breast cancer. Mammary Gland Biol Neoplasia 11:3-11

23. Polychronis A, Dudley Sinnett H, Hadjiminas D et al (2005) Preoperative gefitinib versus gefitinib and anastrozole in postmenopausal patients with oestrogen-receptor positive and epidermal-growth-factor-receptor-positive primary breast cancer: a double-blind placebo-controlled phase II randomised trial. Lancet Oncol 6:383-391

24. Dowsett M, Smith I, Skene A et al (2006) Biological and clinical outcomes from a phase II placebo-controlled neoadjuvant study of anastrozole alone or with gefitinib in postmenopausal women with ER/PgR+ breast cancer. Proc Am Soc Clin Oncol 24:s6 (abstr 515) 